\title{
Macronutrient, Vitamin, and Mineral Intakes in the EPIC-Germany Cohorts
}

\author{
Matthias B. Schulze ${ }^{a}$ Jakob Linseisen ${ }^{b}$ Anja Kroke ${ }^{a}$ Heiner Boeing ${ }^{a}$ \\ a Department of Epidemiology, German Institute of Human Nutrition, Potsdam-Rehbrücke, and \\ ${ }^{b}$ Division of Clinical Epidemiology, German Cancer Research Centre, Heidelberg, Germany
}

\begin{abstract}
This article presents intakes of nutrients in the EPIC-Heidelberg and the EPIC-Potsdam (European Investigation into Cancer and Nutrition) studies. Estimates are based on standardized 24-hour dietary recalls. Recalls from 1,013 men and 1,078 women in Heidelberg and from 1,032 men and 898 women in Potsdam were included in the analysis. The estimated nutrient intake was based on the German Food Code and Nutrient Data Base version II.3. Analyses were carried out stratified by sex and weighted for the day of the week and age. Men in Potsdam reported significantly higher intakes of energy (mean Potsdam $=10,718 \mathrm{~kJ}$, mean Heidelberg $=10,387$ $\mathrm{kJ}$ ) and higher intakes of vitamins and minerals as compared with men in Heidelberg. However, Heidelberg men consumed more alcohol, $\alpha$-tocopherol, phosphorus, calcium, and magnesium. Potsdam women reported lower energy (mean Potsdam $=7,537 \mathrm{~kJ}$, mean Heidelberg $=$ $7,855 \mathrm{~kJ})$, alcohol, and cholesterol intakes as compared
\end{abstract}

EPIC Study, European Prospective Investigation into Cancer and Nutrition - German Part has been published in Annals of Nutrition and Metabolism Vol. 43, No. 4, 1999. with Heidelberg women. Vitamin and mineral intakes were lower too, except for retinol and ascorbic acid. The intakes of energy and most nutrients observed in the Potsdam and Heidelberg study populations were within the range reported from other German studies. The observed differences between both study populations indicate different dietary patterns, increasing the exposure variation in the EPIC study.

\section{Introduction}

Two German cohorts, one in Potsdam and one in Heidelberg, contribute to the EPIC multicenter cohort study which consists of about 475,000 study participants from nine different European countries (Denmark, France, Germany, Great Britain, Greece, Italy, The Netherlands, Sweden, and Spain) [1, 2]. EPIC has been implemented to further elucidate the association between diet and chronic disease, particularly cancer. Country-specific dietary assessment tools were applied to obtain dietary information in EPIC [3]. To overcome methodological differences between centers, the dietary assessment methods were calibrated. As calibration method, 24-hour dietary recalls were selected and applied to a representative subsample of each EPIC study population. The intake estimates from the 24-hour dietary recalls can be used to describe and compare quantitatively the diet of the different EPIC 
cohorts as well as to compare the EPIC cohorts with the general population and other study populations. In a recent analysis, both German cohorts revealed significant differences in food intake [4] that might lead to differences in nutrient intake. This article describes the intakes of macronutrients, vitamins, and minerals in the German EPIC cohorts, as assessed by 24-hour dietary recalls.

\section{Subjects and Methods}

Baseline assessments of the EPIC-Heidelberg and the EPIC-Potsdam cohorts, including a dietary assessment with a self-administered food frequency questionnaire, a computerized lifestyle interview, and anthropometric measurements, were carried out between June 1994 and October 1998 in Heidelberg and between August 1994 and September 1998 in Potsdam [2]. Additionally, standardized computerized 24-hour dietary recalls were applied to a subsample (about 7\%) of each study population between June 1996 and October 1998 in Heidelberg and between Junc 1996 and April 1998 in Potsdam. During this time period 2,121 recalls ( 1,033 from men, 1,088 from women) were obtained in Heidelberg, and 2,173 recalls (1,163 from men, 1,010 from women) were obtained in Potsdam, which represented about $91 \%$ of those approached in Heidelberg and $95 \%$ of those approached in Potsdam. The 24-hour dietary recall method, called EPIC-SOFT [5, 6], was developed as a calibration instrument by the International Agency for Research on Cancer in co-operation with all EPIC study centers to allow international data comparison within EPIC [3].

To enhance the comparability between the two study centers, we included only men aged 40-64 years and women aged 35-64 years in the current analysis. Therefore, 3 men and 1 woman in Heidelberg and 56 men and 7 women in Potsdam were excluded from further analysis. In addition, because only a few recalls were obtained on Fridays in Heidelberg, all Friday recalls were excluded (16 men and 9 women in Heidelberg, 75 men and 105 women in Potsdam), leaving 1,013 men and 1,078 women from Heidelberg and 1,032 men and 898 women from Potsdam for analysis.

The recalls in the Heidelberg and Potsdam study centers were carried out through face-to-face interviews by trained interviewers [6]. The consumed foods were recorded chronologically for the previous day. It was also recorded whether the recorded day represented a common day or was influenced by specific circumstances, like sickness or holidays [7]. The recorded food intake was coded, and the nutrient intake was calculated according to the German Food Code and Nutrient Data Base [8] version II.3. Niacin and folate equivalents were defined according to recent publications, the Dietary Reference Intakes of the USA [9] and the German References for Nutrient Intake [10]. Niacin equivalents represent the sum of nicotinamide, nicotinic acid, and tryptophan (where $60 \mathrm{mg}$ tryptophan $=$ $1 \mathrm{mg}$ niacin equivalents). Folate equivalents represent the sum of food folate and folate from supplements (with $1 \mu \mathrm{g}$ folate equivalents $=1 \mu \mathrm{g}$ food folate $=0.5 \mu \mathrm{g}$ folate from supplements), but since this study was based on food intake only, folate equivalents equal the sum of food folatc. Tocopherol intake was presented as tocopherol equivalents, following the German References for Nutrient Intake [10], and as $\alpha$-tocopherol, following the US Dietary Reference Intakes [11].
All anthropometric measurements followed standardized procedures [12]. Body height was measured to the nearest millimeter and body weight to the nearest $100 \mathrm{~g}$ using a digital scale, and body mass index (BMI) was calculated as body weight in $\mathrm{kg}$ divided by the square of body height in $\mathrm{m}^{2}$. Basal metabolic rate (BMR) was calculated based on the formulas of Schofield [13]. Energy intake was then divided by the estimated BMR, obtaining a measure of relative energy intake that accounted for body weight and age.

Mean, standard deviation, minimum and maximum, as well as 10th, 25th, 50th, 75th, and 90th percentiles were calculated for each study center stratified by sex. All descriptive measures for nutrient intake were adjusted for the day of the week recorded and the age of the participants. The age distribution of the general German population for 1996 provided by the Federal Statistical Office [14] was taken as standard.

The significance of differences between the Potsdam and Heidelberg study was tested using Student's two-sided t test for independent samples and the Mann-Whitney two-sided non-parametric test. Calculation of the weighting factor was performed using the SAS System for Windows (release 8.00; SAS Institute, Cary, N.C., USA). Calculation of descriptive measures weighted for age and weekday and significance tests were performed using SPSS for Windows (release 8.0.0; SPSS, Chicago, Ill., USA).

\section{Results}

The distribution of study subjects and the general German population according to age categories is shown in table 1. Potsdam men and women tended to be slightly older than their Heidelberg counterparts, supporting the need for age adjustment procedures for direct comparison of centers. Also, differences were seen for the distribution of recalls according to day of the week (table 2). Saturdays and Sundays were underrepresented in both centers, and Tuesday was overrepresented. Additionally, small differences in the distribution of days of the week existed between the two study centers. The weighting procedure also eliminated these differences.

The intakes of energy and nutrients were presented as mean, standard deviation, minimum, maximum, and 10th, 25th, 50th, 75th, and 90th percentiles per day for both study populations and by sex. In addition, the data for age, height, weight, BMI, and the ratio of energy intake to BMR were presented. Table 3 shows the data for men. Men in the Potsdam study reported a significantly higher energy intake than men in the Heidelberg study. The contributions of carbohydrates, protein, and alcohol to the total energy intake were higher in Heidelberg men than in Potsdam men, while the opposite was found for the contribution of fat to total energy. Excluding energy from alcohol in the calculation of total energy, the mean contributions of carbohydrates, protein, and fat to the total energy intake were $41.4,15.0$, and $43.6 \%$ in Potsdam 
Table 1. Percentages of men and women according to age categories in the EPIC-Potsdam study, the EPIC-Heidelberg study, and the population of the Federal Republic of Germany (FRG), December 31, 1996

\begin{tabular}{llllllll}
\hline $\begin{array}{l}\text { Age } \\
\text { years }\end{array}$ & Men & \multicolumn{5}{c}{ Women } \\
\cline { 2 - 3 } & $\begin{array}{l}\text { Potsdam } \\
(\mathrm{n}=1,032)\end{array}$ & $\begin{array}{l}\text { Heidelberg } \\
(\mathrm{n}=1,013)\end{array}$ & $\begin{array}{l}\text { FRG 1996 } \\
(\mathrm{n}=13,341,500)\end{array}$ & $\begin{array}{l}\text { Potsdam } \\
(\mathrm{n}=898)\end{array}$ & $\begin{array}{l}\text { Heidelberg } \\
(\mathrm{n}=1,078)\end{array}$ & $\begin{array}{l}\text { FRG 1996 } \\
(\mathrm{n}=16,457,200)\end{array}$ \\
\hline $35-39$ & - & - & - & 10.4 & 16.0 & 19.6 \\
$40-44$ & 10.6 & 13.6 & 22.3 & 8.1 & 16.1 & 17.5 \\
$45-49$ & 12.9 & 20.8 & 20.2 & 15.0 & 14.7 & 16.0 \\
$50-54$ & 14.5 & 18.4 & 17.6 & 13.4 & 16.2 & 13.9 \\
$55-59$ & 30.8 & 21.7 & 22.7 & 26.8 & 19.9 & 18.4 \\
$60-64$ & 31.2 & 25.5 & 17.2 & 26.3 & 17.2 & 14.6 \\
\hline
\end{tabular}

Table 2. Percentages of men and women according to the day of the week the recalls were obtained in the EPIC-Potsdam and the EPIC-Heidelberg studies

\begin{tabular}{|c|c|c|c|c|}
\hline \multirow[t]{2}{*}{ Day of the week } & \multicolumn{2}{|l|}{ Men } & \multicolumn{2}{|l|}{ Women } \\
\hline & $\begin{array}{l}\text { Potsdam } \\
(\mathrm{n}=1,032)\end{array}$ & $\begin{array}{l}\text { Heidelberg } \\
(\mathrm{n}=1,013)\end{array}$ & $\begin{array}{l}\text { Potsdam } \\
(\mathrm{n}=898)\end{array}$ & $\begin{array}{l}\text { Heidelberg } \\
(\mathrm{n}=1,078)\end{array}$ \\
\hline Monday & 20.7 & 23.0 & 18.6 & 22.0 \\
\hline Tuesday & 22.3 & 21.3 & 23.8 & 22.0 \\
\hline Wednesday & 19.4 & 17.6 & 18.5 & 17.5 \\
\hline Thursday & 14.0 & 14.6 & 13.4 & 14.8 \\
\hline Saturday & 13.6 & 11.2 & 11.8 & 11.1 \\
\hline Sunday & 10.1 & 12.3 & 13.9 & 12.5 \\
\hline
\end{tabular}

men and 44.0, 16.2, and 39.8\% in Heidelberg men. While absolute carbohydrate and protein intakes were similar, the fat intake in Potsdam men exceeded that of Heidelberg men. Alcohol intake was estimated to be higher in men from Heidelberg than in men from Potsdam. Mean and median fiber intakes were similar; however, the variation of intake was higher in Heidelberg than in Potsdam. With regard to vitamin intake, men of the Potsdam cohort reported higher intakes of retinol, vitamin $\mathrm{D}$, ascorbic acid, thiamine, vitamin $\mathrm{B}_{6}$, vitamin $\mathrm{B}_{12}$, and tocopherol equivalents, while men of the Heidelberg cohort reported higher intakes of $\alpha$-tocopherol. No significant differences were found for $\beta$-carotene, riboflavin, niacin, and folate intakes. Phosphorus, calcium, magnesium, and zinc intakes were found to be higher in Heidelberg men than in Potsdam men. Potassium and iron intakes were not significantly different.

With respect to the energy intake in women, women of the Heidelberg cohort reported a significantly higher intake as compared with women of the Potsdam cohort (table 4). The contribution of carbohydrates to the total energy intake was found to be higher in Potsdam women as compared with Heidelberg women, while the opposite was observed for protein and alcohol. Excluding energy from alcohol in the calculation of total energy, the mean contributions of carbohydrates, protein, and fat to the total energy intake were $46.0,14.5$, and $39.5 \%$, respectively, in Potsdam women and 45.6, 15.2, and $39.2 \%$, respectively, in Heidelberg women. Here, only the contribution of protein was found to be significantly different. While absolute carbohydrate and fat intakes were not different, the protein intake in Heidelberg women exceeded that of Potsdam women. Alcohol and cholesterol intakes were found to be higher in Heidelberg; fiber intake was not significantly different between centers. The intakes of $\beta$-carotene, tocopherol equivalents, $\alpha$-tocopherol, riboflavin, niacin, folate, and vitamin $\mathrm{B}_{12}$ were significantly higher in Heidelberg women, while women in Potsdam reported higher intakes of retinol and ascorbic acid. Mineral intake was generally higher in Heidelberg with the exception of potassium, for which no significantly different intakes were observed. 


\section{Discussion}

This paper reports quantitative estimates of energy and nutrient intakes from the two German EPIC cohorts. Data are given as mean, standard deviation, and percentiles including median in order to allow a detailed data evaluation. Major differences between both study centers were found regarding the intakes of energy, fat, protein, alcohol, and most vitamins and minerals.

Since for most nutrients the intake is closely linked to total energy intake [15], differences in energy intakes between both study centers have consequently led to dif-

Table 3. Mean, standard deviation, minimum, maximum, and selected percentiles of age, height, weight, BMI, and daily intakes of energy and nutrients in men; results from 24-hour dietary recalls, EPIC-Potsdam and EPIC-Heidelberg studies

\begin{tabular}{|c|c|c|c|c|c|c|c|c|c|}
\hline \multirow[t]{3}{*}{ Variables } & \multicolumn{9}{|c|}{ Potsdam $(\mathrm{n}=1,032)$} \\
\hline & \multirow[t]{2}{*}{ mean } & \multirow[t]{2}{*}{ SD } & \multirow{2}{*}{$\begin{array}{l}\text { mini- } \\
\text { mum }\end{array}$} & \multirow{2}{*}{$\begin{array}{l}\text { maxi- } \\
\text { mum }\end{array}$} & \multicolumn{5}{|c|}{ percentiles } \\
\hline & & & & & 10 & 25 & 50 & 75 & 90 \\
\hline \multicolumn{10}{|l|}{ Age and anthropometry } \\
\hline Age, years ${ }^{\mathrm{a}}$ & 55 & 7 & 40 & 64 & 45 & 50 & 57 & 61 & 63 \\
\hline Height, $\mathrm{cm}^{\mathrm{a}}$ & 175 & 7 & 151 & 196 & 166 & 170 & 175 & 179 & 183 \\
\hline Weight, kg & 83 & 12 & 50 & 148 & 70 & 75 & 82 & 90 & 99 \\
\hline $\mathrm{BMI}^{\mathrm{a}}$ & 27.2 & 3.6 & 17.8 & 48.9 & 23.1 & 24.9 & 26.8 & 29.3 & 31.7 \\
\hline Ratio total energy intake:BMR ${ }^{\mathrm{a}}$ & 1.44 & 1.39 & 0.24 & 3.67 & 0.88 & 1.11 & 1.39 & 1.71 & 2.06 \\
\hline \multicolumn{10}{|l|}{ Daily energy and nutrient intakes ${ }^{b}$} \\
\hline Total encrgy, kJ & 10,718 & 3,550 & 1,191 & 27,429 & 6,663 & 8,279 & 10,438 & 12,388 & 15,513 \\
\hline Carbohydrate contribution, $\% \mathrm{a}^{\mathrm{a}, \mathrm{c}}$ & 38.8 & 9.5 & 8.4 & 89.0 & 27.8 & 32.3 & 37.8 & 44.0 & 51.7 \\
\hline Protein contribution, $\% \mathrm{a}^{\mathrm{a}, \mathrm{c}}$ & 14.0 & 3.9 & 2.0 & 36.4 & 9.8 & 11.3 & 13.4 & 16.2 & 19.2 \\
\hline Fat contribution, $\% \mathrm{a}, \mathrm{c}$ & 40.9 & 9.4 & 4.1 & 65.8 & 29.4 & 34.7 & 41.2 & 47.5 & 53.0 \\
\hline Alcohol contribution, $\%{ }^{a, c}$ & 6.3 & 7.6 & 0.0 & 76.8 & 0.0 & 0.0 & 4.5 & 10.1 & 16.0 \\
\hline Carbohydrates, g & 242.2 & 87.8 & 21.8 & 709.1 & 140.6 & 181.7 & 232.4 & 289.1 & 354.3 \\
\hline Protein, $\mathrm{g}$ & 88.0 & 36.8 & 11.4 & 367.0 & 49.3 & 63.9 & 82.9 & 104.7 & 132.0 \\
\hline Fat, $\mathrm{g}^{\mathrm{a}, \mathrm{c}}$ & 116.8 & 51.5 & 7.7 & 388.2 & 58.7 & 81.2 & 111.1 & 146.7 & 179.1 \\
\hline Alcohol, ga, c & 24.3 & 30.9 & 0.0 & 245.7 & 0.0 & 0.0 & 19.8 & 39.7 & 60.4 \\
\hline Cholesterol, mg & 377 & 225 & 10 & 1,410 & 140 & 210 & 320 & 500 & 690 \\
\hline Fiber, $g^{c}$ & 21.9 & 9.2 & 1.4 & 65.2 & 11.5 & 15.5 & 20.5 & 27.0 & 33.9 \\
\hline Retinol, mgc & 1.2 & 2.1 & 0.0 & 57.6 & 0.3 & 0.5 & 0.7 & 1.3 & 2.5 \\
\hline$\beta$-Carotene, mg & 2.9 & 3.2 & 0.1 & 50.4 & 0.7 & 1.2 & 2.0 & 3.4 & 5.9 \\
\hline Vitamin $\mathrm{D}, \mu \mathrm{g}^{\mathrm{a}, \mathrm{c}}$ & 4.6 & 8.2 & 0.0 & 104.0 & 0.8 & 1.4 & 2.5 & 4.2 & 8.2 \\
\hline Tocopherol equivalents, $\mathrm{mg}^{\mathrm{c}}$ & 15.0 & 9.1 & 0.9 & 62.7 & 5.7 & 8.9 & 12.9 & 19.3 & 26.1 \\
\hline$\alpha$-Tocopherol, mga,b & 9.8 & 7.4 & 0.2 & 54.0 & 3.4 & 4.9 & 7.6 & 11.9 & 18.4 \\
\hline Ascorbic acid, mga, c & 131 & 110 & 0 & 1,676 & 34 & 59 & 103 & 171 & 256 \\
\hline Thiamine, $\mathrm{mg}^{\mathrm{a}, \mathrm{c}}$ & 1.5 & 0.7 & 0.1 & 7.2 & 0.7 & 1.0 & 1.3 & 1.8 & 2.4 \\
\hline Riboflavin, mg & 1.7 & 0.7 & 0.2 & 11.1 & 1.0 & 1.2 & 1.6 & 2.1 & 2.6 \\
\hline Niacin equivalents, mg & 36.7 & 14.8 & 7.1 & 118.2 & 21.0 & 26.8 & 34.0 & 43.0 & 56.0 \\
\hline Vitamin $\mathrm{B}_{6}, \mathrm{mg}^{\mathrm{a}, \mathrm{c}}$ & 1.9 & 0.8 & 0.1 & 6.8 & 1.1 & 1.4 & 1.8 & 2.3 & 2.9 \\
\hline Dietary folate equivalents, $\mu \mathrm{g}$ & 238 & 101 & 23 & 1,279 & 134 & 171 & 226 & 288 & 350 \\
\hline Vitamin $\mathrm{B}_{12}, \mu \mathrm{g}^{\mathrm{c}}$ & 8 & 6 & 0 & 118 & 3 & 4 & 6 & 10 & 13 \\
\hline Potassium, g & 3.4 & 1.1 & 0.5 & 9.1 & 2.1 & 2.5 & 3.2 & 4.0 & 4.8 \\
\hline Phosphorus, ga, c & 1.4 & 0.5 & 0.2 & 3.6 & 0.8 & 1.1 & 1.3 & 1.7 & 2.0 \\
\hline Calcium, mga, c & 774 & 414 & 80 & 2,493 & 334 & 480 & 689 & 963 & 1,330 \\
\hline Magnesium, mga, c & 371 & 125 & 57 & 1,164 & 241 & 290 & 349 & 434 & 532 \\
\hline Iron, $\mathrm{mg}$ & 14.6 & 5.7 & 2.2 & 52.2 & 8.7 & 10.9 & 13.4 & 17.0 & 21.6 \\
\hline Zinc, mga, c & 12.0 & 4.6 & 1.3 & 32.1 & 7.0 & 8.7 & 11.2 & 14.4 & 18.3 \\
\hline
\end{tabular}

Significantly different means between Potsdam and Heidelberg $(\mathrm{p}<0.05$, two-sided) using Student's $\mathrm{t}$ test for independent samples.

Adjusted for age (according to the population of the Federal Republic of Germany, December 31, 1996) and day of the week. 
ferences in nutrient intakes as well. While this might explain that women in Heidelberg had higher intakes of most vitamins and minerals, Heidelberg men had higher intakes of several nutrients despite their lower energy intakes as compared with Potsdam men. Differences of relative nutrient intake, after adjustment for energy, would in these cases be even higher. It is, however, likely that the differences in energy and nutrient intakes observed between Potsdam and Heidelberg might partly be due to differential underreporting. Underestimation of energy intake was found to be related to obesity and affected the relation of macronutrients according to re-

\begin{tabular}{|c|c|c|c|c|c|c|c|c|}
\hline \multicolumn{9}{|c|}{ Heidelberg $(\mathrm{n}=1,013)$} \\
\hline \multirow[t]{2}{*}{ mean } & \multirow[t]{2}{*}{ SD } & \multirow{2}{*}{$\begin{array}{l}\text { mini- } \\
\text { mum }\end{array}$} & \multirow{2}{*}{$\begin{array}{l}\text { maxi- } \\
\text { mum }\end{array}$} & \multicolumn{5}{|c|}{ percentiles } \\
\hline & & & & 10 & 25 & 50 & 75 & 90 \\
\hline 52 & 7 & 40 & 64 & 42 & 46 & 52 & 58 & 62 \\
\hline 176 & 7 & 154 & 196 & 168 & 172 & 176 & 180 & 185 \\
\hline 83 & 13 & 51 & 130 & 69 & 74 & 82 & 91 & 100 \\
\hline 26.7 & 3.7 & 17.1 & 40.1 & 22.5 & 24.3 & 26.3 & 28.7 & 31.6 \\
\hline 1.39 & 1.34 & 0.20 & 4.85 & 0.82 & 1.05 & 1.35 & 1.68 & 1.99 \\
\hline 10,387 & 3,806 & 1,451 & 34,683 & 6,120 & 7,856 & 9,980 & 12,437 & 14,889 \\
\hline 40.6 & 10.2 & 10.0 & 80.4 & 28.3 & 33.9 & 40.0 & 46.7 & 53.8 \\
\hline 14.8 & 4.1 & 6.4 & 55.3 & 10.3 & 12.1 & 14.1 & 17.0 & 20.0 \\
\hline 36.6 & 9.3 & 8.2 & 70.6 & 24.6 & 30.2 & 37.1 & 43.2 & 48.0 \\
\hline 7.9 & 8.4 & 0.0 & 50.8 & 0.0 & 0.0 & 6.0 & 13.1 & 19.6 \\
\hline 247.3 & 107.4 & 32.3 & $1,086.4$ & 130.7 & 175.7 & 231.9 & 303.7 & 382.4 \\
\hline 89.9 & 39.7 & 6.8 & 493.7 & 49.1 & 63.7 & 83.5 & 109.1 & 134.5 \\
\hline 100.9 & 48.1 & 5.8 & 421.7 & 46.8 & 67.4 & 94.3 & 129.3 & 160.8 \\
\hline 28.6 & 31.8 & 0.0 & 226.6 & 0.0 & 0.0 & 20.5 & 43.8 & 71.1 \\
\hline 371 & 226 & 0 & 1,590 & 140 & 210 & 330 & 480 & 672 \\
\hline 21.8 & 11.5 & 0.8 & 106.5 & 10.2 & 14.0 & 19.9 & 26.9 & 35.0 \\
\hline 1.0 & 3.3 & 0.0 & 74.6 & 0.2 & 0.3 & 0.5 & 0.8 & 1.6 \\
\hline 3.2 & 4.6 & 0.0 & 73.9 & 0.5 & 1.1 & 2.0 & 3.8 & 6.6 \\
\hline 3.5 & 7.2 & 0.0 & 96 & 0 & 1 & 2 & 3 & 6 \\
\hline 14.3 & 10.1 & 0.9 & 221.3 & 5.1 & 8.2 & 12.2 & 18.2 & 25.2 \\
\hline 12.0 & 9.6 & 0.8 & 217.2 & 3.8 & 6.1 & 9.9 & 15.5 & 22.1 \\
\hline 120 & 101 & 0 & 1,135 & 31 & 51 & 93 & 153 & 259 \\
\hline 1.4 & 0.7 & 0.1 & 7.5 & 0.6 & 0.9 & 1.3 & 1.7 & 2.3 \\
\hline 1.7 & 0.9 & 0.2 & 15.2 & 0.9 & 1.2 & 1.6 & 2.0 & 2.5 \\
\hline 36.1 & 15.2 & 3.3 & 164.0 & 20.4 & 25.5 & 33.8 & 44.0 & 55.4 \\
\hline 1.8 & 0.8 & 0.2 & 10.4 & 1.0 & 1.3 & 1.7 & 2.2 & 2.8 \\
\hline 245 & 119 & 24 & 1,160 & 125 & 169 & 229 & 301 & 376 \\
\hline 7 & 11 & 0 & 276 & 2 & 4 & 5 & 8 & 13 \\
\hline 3.3 & 1.3 & 0.8 & 13.3 & 1.9 & 2.5 & 3.2 & 4.0 & 4.8 \\
\hline 1.5 & 0.5 & 0.2 & 5.2 & 0.8 & 1.1 & 1.4 & 1.8 & 2.1 \\
\hline 885 & 485 & 105 & 3,897 & 369 & 529 & 787 & 1,137 & 1,494 \\
\hline 387 & 143 & 93 & 1,570 & 228 & 292 & 368 & 459 & 558 \\
\hline 15.1 & 6.9 & 3.2 & 79.1 & 8.4 & 10.8 & 14.1 & 17.8 & 22.1 \\
\hline 12.7 & 5.1 & 2.1 & 43.3 & 7.4 & 9.3 & 12.0 & 15.2 & 18.8 \\
\hline
\end{tabular}

c Significantly different distributions between Potsdam and Heidelberg ( $p<0.05$, twosided) using the Mann-Whitney non-parametric test. 
cent analyses of data from the food frequency questionnaire applied in EPIC-Germany [16] as well as of data from the 24-hour dietary recalls [17]. Differential underreporting is indicated by the different ratios between energy intake and BMR observed, particularly for women. Energy intake was significantly lower in Potsdam women despite the significantly higher weight and BMI. However, whether this observation reflects differential underreporting or rather reflects lower energy needs in Potsdam women due to less physical activity cannot be easily distinguished, since reliable data on energy expenditure are not available. Several methodological steps should have

Table 4. Mean, standard deviation, minimum, maximum, and selected percentiles of age, height, weight, BMI, and daily intakes of energy and nutrients in women; results from 24-hour dietary recalls, EPIC-Potsdam and EPIC-Heidelberg studies

\begin{tabular}{|c|c|c|c|c|c|c|c|c|c|}
\hline \multirow[t]{3}{*}{ Variables } & \multicolumn{9}{|c|}{ Potsdam $(n=898)$} \\
\hline & \multirow[t]{2}{*}{ mean } & \multirow[t]{2}{*}{ SD } & \multirow{2}{*}{$\begin{array}{l}\text { mini- } \\
\text { mum }\end{array}$} & \multirow{2}{*}{$\begin{array}{l}\operatorname{maxi}- \\
\text { mum }\end{array}$} & \multicolumn{5}{|c|}{ percentiles } \\
\hline & & & & & 10 & 25 & 50 & 75 & 90 \\
\hline \multicolumn{10}{|l|}{ Age and anthropometry } \\
\hline Age, years ${ }^{a}$ & 53 & 8 & 35 & 64 & 39 & 47 & 55 & 60 & 62 \\
\hline Height, $\mathrm{cm}^{\mathrm{a}}$ & 162 & 6 & 142 & 190 & 154 & 158 & 163 & 167 & 170 \\
\hline Weight, $\mathrm{kg}^{\mathrm{a}}$ & 70 & 13 & 41 & 138 & 55 & 60 & 68 & 76 & 87 \\
\hline $\mathrm{BMI}^{\mathrm{a}}$ & 26.4 & 4.6 & 15.8 & 46.5 & 21.3 & 25.6 & 25.6 & 29.0 & 32.8 \\
\hline Ratio total energy intake:BMR ${ }^{\mathrm{a}}$ & 1.30 & 1.25 & 0.13 & 4.59 & 0.80 & 1.01 & 1.25 & 1.55 & 1.88 \\
\hline \multicolumn{10}{|l|}{ Daily energy and nutrient intakes ${ }^{b}$} \\
\hline Total energy, kJ ${ }^{\mathrm{a}, \mathrm{c}}$ & 7,537 & 2,568 & 745 & 24,668 & 4,658 & 5,841 & 7,225 & 8,918 & 10,900 \\
\hline Carbohydrate contribution, $\%^{a, c}$ & 44.4 & 10.8 & 15.3 & 90.4 & 31.0 & 37.1 & 43.9 & 51.0 & 58.1 \\
\hline Protein contribution, $\% \mathrm{a}^{\mathrm{a}, \mathrm{c}}$ & 13.9 & 4.2 & 3.1 & 60.2 & 9.5 & 11.3 & 13.3 & 15.9 & 19.0 \\
\hline Fat contribution, $\%$ & 38.1 & 9.6 & 6.4 & 70.5 & 25.6 & 31.8 & 38.4 & 44.4 & 49.9 \\
\hline Alcohol contribution, $\%$ a, c & 3.6 & 6.1 & 0.0 & 48.3 & 0.0 & 0.0 & 0.0 & 5.6 & 11.5 \\
\hline Carbohydrates, g & 196.1 & 77.9 & 9.8 & 697.3 & 107.3 & 145.0 & 189.0 & 235.7 & 294.0 \\
\hline Protein, ga, c & 60.9 & 24.6 & 4.7 & 173.5 & 32.5 & 43.8 & 58.3 & 74.8 & 92.4 \\
\hline Fat, $\mathrm{g}$ & 76.1 & 34.5 & 3.4 & 263.0 & 36.9 & 51.9 & 73.1 & 94.4 & 120.9 \\
\hline Alcohol, ga, c & 9.7 & 16.4 & 0.0 & 97.4 & 0.0 & 0.0 & 0.1 & 13.2 & 31.5 \\
\hline Cholesterol, mga, c & 264 & 177 & 0 & 1,120 & 90 & 140 & 220 & 350 & 510 \\
\hline Fiber, $\mathrm{g}$ & 19.4 & 8.2 & 1.3 & 58.6 & 9.6 & 13.7 & 18.2 & 24.1 & 30.1 \\
\hline Retinol, mgc & 0.7 & 1.1 & 0.0 & 22.3 & 0.2 & 0.3 & 0.5 & 0.7 & 1.3 \\
\hline$\beta$-Carotene, $\mathrm{mg}^{\mathrm{c}}$ & 3.6 & 6.7 & 0.0 & 119.8 & 0.6 & 1.1 & 1.9 & 3.6 & 7.6 \\
\hline Vitamin $\mathrm{D}, \mu \mathrm{g}$ & 3.1 & 5.6 & 0 & 50.5 & 0.0 & 0.1 & 1.7 & 3.0 & 5.1 \\
\hline Tocopherol equivalents, $\mathrm{mg}^{\mathrm{a}}$ & 11.3 & 6.9 & 0.2 & 67.3 & 4.9 & 6.5 & 9.5 & 14.3 & 19.5 \\
\hline$\alpha$-Tocopherol, mga,c & 8.3 & 6.1 & 0.2 & 48.9 & 3.0 & 4.3 & 6.5 & 10.3 & 15.3 \\
\hline Ascorbic acid, $\mathrm{mg}^{\mathrm{c}}$ & 130 & 103 & 0 & 983 & 32 & 60 & 102 & 168 & 257 \\
\hline Thiamine, $\mathrm{mg}$ & 1.0 & 0.5 & 0.2 & 3.3 & 0.5 & 0.7 & 0.9 & 1.3 & 1.6 \\
\hline Riboflavin, ma ${ }^{\mathrm{a}, \mathrm{c}}$ & 1.3 & 0.6 & 0.1 & 5.0 & 0.7 & 0.9 & 1.2 & 1.6 & 2.1 \\
\hline Niacin equivalents, $\mathrm{mg}^{\mathrm{a}, \mathrm{c}}$ & 24.7 & 9.6 & 2.3 & 86.2 & 14.6 & 17.9 & 23.2 & 29.7 & 36.8 \\
\hline Vitamin $\mathrm{B}_{6}, \mathrm{mg}$ & 1.4 & 0.6 & 0.1 & 4.5 & 0.7 & 1.0 & 1.3 & 1.7 & 2.2 \\
\hline Dietary folate equivalents, $\mu \mathrm{g}^{\mathrm{a}, \mathrm{c}}$ & 196 & 85 & 8 & 815 & 99 & 138 & 183 & 242 & 312 \\
\hline Vitamin $\mathrm{B}_{12}, \mu \mathrm{g}^{\mathrm{a}}$ & 4 & 4 & 0 & 48 & 1 & 2 & 4 & 6 & 9 \\
\hline Potassium, $\mathrm{g}$ & 2.8 & 1.0 & 0.2 & 13.3 & 1.7 & 2.1 & 2.7 & 3.4 & 4.0 \\
\hline Phosphorus, ga, c & 1.0 & 0.4 & 0.1 & 3.1 & 0.6 & 0.8 & 1.0 & 1.3 & 1.6 \\
\hline Calcium, mga, c & 707 & 387 & 67 & 2,540 & 287 & 440 & 633 & 903 & 1,225 \\
\hline Magnesium, mga, c & 289 & 105 & 28 & 1,316 & 173 & 217 & 279 & 344 & 404 \\
\hline Iron, $\mathrm{mg}^{\mathrm{a}, \mathrm{c}}$ & 11.6 & 4.3 & 2.0 & 46.3 & 6.9 & 8.5 & 11.0 & 13.7 & 17.2 \\
\hline Zinc, mga, c & 9.0 & 3.3 & 1.0 & 27.3 & 5.5 & 6.7 & 8.5 & 11.0 & 13.3 \\
\hline
\end{tabular}

a Significantly different means between Potsdam and Heidelberg $(\mathrm{p}<0.05$, two-sided) using Student's $\mathrm{t}$ test for independent samples.

Adjusted for age (according to the population of the Federal Republic of Germany, December 31, 1996) and day of the week. 
ensured a high comparability of estimates between both centers. The dietary data collections in the two German cohorts were conducted during comparable time periods with the same dietary assessment instrument and under a common study protocol and interviewer training. Coding procedures and data analyses were also identical, and the analyses were based on data adjusted for age of the study subjects and the day of the week. However, an outlying interviewer in Heidelberg explains most of the differences between interviewers when nested by center [18]. An interviewer effect, explaining in part the observed differences, cannot be excluded despite these attempts for stan-

\begin{tabular}{|c|c|c|c|c|c|c|c|c|}
\hline \multicolumn{9}{|c|}{ Heidelberg $(n=1,013)$} \\
\hline \multirow[t]{2}{*}{ mean } & \multirow[t]{2}{*}{ SD } & \multirow{2}{*}{$\begin{array}{l}\text { mini- } \\
\text { mum }\end{array}$} & \multirow{2}{*}{$\begin{array}{l}\text { maxi- } \\
\text { mum }\end{array}$} & \multicolumn{5}{|c|}{ percentiles } \\
\hline & & & & 10 & 25 & 50 & 75 & 90 \\
\hline 50 & 9 & 35 & 64 & 38 & 42 & 52 & 58 & 61 \\
\hline 164 & 6 & 145 & 194 & 156 & 160 & 164 & 168 & 172 \\
\hline 68 & 13 & 40 & 123 & 54 & 59 & 66 & 75 & 84 \\
\hline 25.2 & 4.8 & 16.1 & 47.1 & 20.2 & 21.8 & 24.2 & 27.5 & 31.6 \\
\hline 1.35 & 1.30 & 0.22 & 4.31 & 0.79 & 1.03 & 1.30 & 1.59 & 1.94 \\
\hline 7,855 & 2,761 & 1,231 & 24,048 & 4,657 & 5,979 & 7,544 & 9,307 & 11,468 \\
\hline 43.5 & 10.2 & 11.2 & 95.3 & 31.0 & 36.4 & 43.1 & 50.2 & 56.4 \\
\hline 14.5 & 4.2 & 2.4 & 34.7 & 9.8 & 11.7 & 14.0 & 16.5 & 19.7 \\
\hline 37.3 & 9.1 & 2.3 & 70.2 & 26.0 & 31.4 & 37.5 & 43.3 & 48.5 \\
\hline 4.8 & 6.6 & 0.0 & 43.2 & 0.0 & 0.0 & 0.4 & 8.5 & 14.0 \\
\hline 198.4 & 76.2 & 41.9 & 720.1 & 112.6 & 145.8 & 188.8 & 239.9 & 295.5 \\
\hline 65.6 & 25.6 & 2.3 & 189.0 & 36.2 & 48.1 & 62.1 & 79.6 & 97.9 \\
\hline 78.0 & 38.0 & 1.0 & 339.6 & 36.5 & 51.1 & 73.6 & 96.5 & 125.5 \\
\hline 13.8 & 20.4 & 0.0 & 189.4 & 0.0 & 0.0 & 1.1 & 22.6 & 40.0 \\
\hline 285 & 185 & 0 & 1,780 & 100 & 160 & 240 & 370 & 520 \\
\hline 19.6 & 9.0 & 1.1 & 70.4 & 10.0 & 13.2 & 18.3 & 23.9 & 30.9 \\
\hline 0.8 & 2.3 & 0.0 & 39.1 & 0.2 & 0.3 & 0.4 & 0.6 & 1.1 \\
\hline 3.7 & 4.8 & 0.0 & 64.8 & 0.6 & 1.2 & 2.2 & 4.4 & 8.0 \\
\hline 2.9 & 5.8 & 0.0 & 107 & 0.0 & 0.8 & 1.5 & 2.8 & 4.8 \\
\hline 12.3 & 9.5 & 0.2 & 173.9 & 4.5 & 6.7 & 10.3 & 15.7 & 22.4 \\
\hline 10.6 & 9.1 & 0.1 & 170.1 & 3.4 & 5.4 & 8.5 & 13.9 & 19.8 \\
\hline 126 & 104 & 0 & 942 & 32 & 55 & 98 & 167 & 259 \\
\hline 1.0 & 0.5 & 0.0 & 4.0 & 0.5 & 0.7 & 1.0 & 1.3 & 1.7 \\
\hline 1.4 & 0.7 & 0.1 & 8.0 & 0.8 & 1.0 & 1.3 & 1.7 & 2.1 \\
\hline 25.9 & 10.0 & 1.0 & 71.3 & 14.6 & 19.2 & 24.5 & 31.1 & 39.3 \\
\hline 1.4 & 0.6 & 0.0 & 6.2 & 0.7 & 1.0 & 1.3 & 1.7 & 2.2 \\
\hline 213 & 97 & 22 & 1,024 & 110 & 146 & 197 & 263 & 332 \\
\hline 5 & 8 & 0 & 140 & 1 & 2 & 4 & 6 & 9 \\
\hline 2.8 & 1.0 & 0.1 & 9.5 & 1.7 & 2.2 & 2.8 & 3.4 & 4.1 \\
\hline 1.2 & 0.4 & 0.0 & 3.4 & 0.7 & 0.9 & 1.1 & 1.4 & 1.7 \\
\hline 837 & 412 & 86 & 3,591 & 385 & 544 & 777 & 1,063 & 1,369 \\
\hline 313 & 105 & 33 & 904 & 190 & 240 & 300 & 372 & 450 \\
\hline 12.3 & 4.7 & 2.5 & 34.3 & 7.2 & 9.0 & 11.6 & 14.6 & 18.0 \\
\hline 10.6 & 11.1 & 0.9 & 267.5 & 6.1 & 7.5 & 9.7 & 12.5 & 15.0 \\
\hline
\end{tabular}

c Significantly different distributions between Potsdam and Heidelberg ( $\mathrm{p}<0.05$, twosided) using the Mann-Whitney non-parametric test. 
dardized assessment and analysis procedures. Methodological differences between study centers have been previously reported to have potentially affected the differences of food intakes observed [4] and might have, therefore, affected differences of nutrient intakes as well. Aspects of these methodological differences are that the study populations were not equally distributed over all seasons, that differences existed regarding the location of interviews, and that the proportion of days not reflecting common days was slightly different in the two populations. The latter might have particularly contributed to a higher intake of alcohol in Heidelberg as compared with Potsdam.

The finding that women consume equal or higher amounts of $\beta$-carotene and ascorbic acid than men, despite their generally lower absolute caloric intake, is consistent with the observed higher intakes of fruits and vegetables in women that have been previously reported from the EPIC-Germany study populations [4]. In addition, similar trends were reported from other dietary studies in Germany [19-23]. The alcohol intake of men exceeded that of women more than twofold, an observation consistently reported from other studies in Germany [19-24]. However, sex-related differences need to be interpreted with caution, since the women in this study tended to be younger than the men, but the weighting procedure eliminated only age differences between centers but not between genders.

The estimates of energy intake agree with those reported from the recently completed German Nutrition Survey (GeNuS) 1998 [24]. However, absolute fat intake was found to be generally higher in the EPIC study populations as compared with the GeNuS population, resulting in a higher contribution of fat to total energy intake, while the opposite was found for carbohydrates and protein. The German MONICA studies performed in Augsburg and Erfurt [20, 25, 26] and the NVS/VERA study [19] reported similar estimates of the recent contribution of macronutrients to energy intake, while Thiel et al. [23] reported a higher proportion of energy from fat in an east German study population as compared with our results. Cholesterol and fiber intakes in the EPIC studies were found to be lower as compared with the results of the GeNuS [24]. The German MONICA studies reported higher cholesterol, but similar fiber intakes $[20,25,26]$. The intakes of most vitamins and minerals observed in the Potsdam and Heidelberg study populations were within the range reported from other German studies [19-21, $25,26]$. However, we observed generally higher ascorbic acid and calcium intakes, resulting most probably from the generally higher estimates of fruit, vegetable, and milk product consumption that has been previously reported [4].

Comparison of the EPIC data with those of other studies might be difficult due to differences in the dietary assessment methods and different age and anthropometric population characteristics. The MONICA studies, the NVS/VERA study, and the GeNuS used quantitative assessment tools, allowing a direct comparison with our data. However, methodological studies on the comparability of assessment methods are rare. While the dietary assessment instrument applied in GeNuS was recently validated using EPIC-SOFT, and while preliminary results suggest a good correspondence between both methods for mean macronutrient intake [24], the estimates of dietary intake in the GeNuS were not weighted for the general German age distribution as in our study. Furthermore, the differential performance of the 24-hour dietary recall method applied in EPIC as compared with other methods might be indicated by the lower mean ratio of energy intake to BMR observed in women in our study as compared with that reported from the NVS/VERA study [27]. Moreover, the number of days being covered in other German studies was generally different as compared with EPIC. Only one 24-hour dietary recall per person was obtained in EPIC, unlikely to reflect true long-term averages for any subject. Rather, the resulting inability to correct estimates for day-to-day within-person variation has most likely resulted in exaggerated variation estimates [15]. This effect might be especially severe for most micronutrients where within-person variance has usually been found to be much greater than between-person variance [15]. Furthermore, the food composition database generally used in German studies has changed considerably over time. Our results were based on the same version as were results reported from the GeNuS [24], but were not compared to other German studies [19-21, 25, 26].

In conclusion, we found reasonable values for macroand micronutrient intakes in the German EPIC cohorts. Some methodological problems remain which need to be considered in future risk analyses using dietary data from the two German EPIC cohorts. The observed differences between both study populations indicate different dietary behaviors, increasing the exposure variation in EPIC. 


\section{Acknowledgements}

We wish to thank all study participants for their co-operation and all interviewers, programmers, and documentarists who devoted their energy to collect and process all the data. The recruitment phase of the EPIC-Potsdam study was mainly supported by the Federal Ministry of Science, Germany (grant No. 01 EA 9401). Further financial support was given by the 'Europe against Cancer' program of the European Community (grant No. SOC 95201408 05F02). The recruitment phase of the EPIC-Heidelberg study was mainly supported by the German Cancer Aid (grant No. 70-2201-Bo2). Both EPIC studies are now supported by the German Cancer Aid (grant No. 70-2488-Ha I) and the European Community (grant No. SI2.195579 05F02). M.B. Schulze was furthermore financially supported by the Deutsche Forschungsgemeinschaft (grant No. BO 807/6-1).

\section{References}

1 Riboli E, Kaaks R: The EPIC Project: Rationale and study design. European Prospective Investigation into Cancer and Nutrition. Int J Epidemiol 1997;26(suppl 1):6-14.

2 Boeing $\mathrm{H}$, Wahrendorf J, Becker N: EPIC-Germany - a source for studies into diet and risk of chronic diseases. Ann Nutr Metab 1999;43: 195-204.

3 Kaaks R, Riboli E: Validation and calibration of dietary intake measurements in the EPIC Project: Methodological considerations. European Prospective Investigation into Cancer and Nutrition. Int J Epidemiol 1997;26(suppl 1):15-25.

4 Schulze MB, Brandstetter BR, Kroke A, Wahrendorf J, Boeing $\mathrm{H}$ : Quantitative food intake in the EPIC-Germany cohorts. Ann Nutr Metab 1999;43:235-245.

5 Slimani N, Deharveng G, Charrondière RU, van Kappel AL, Ocké MC, Welch A, Lagiou A van Liere M, Agudo A, Pala V, Brandstetter B, Andren C, Stripp C, van Staveren WA, Riboli $\mathrm{E}$ : Structure of the standardized computerized 24-h diet recall interview used as reference method in the 22 centers participating in the EPIC Project. European Prospective Investigation into Cancer and Nutrition. Comput Methods Programs Biomed 1999;58:251-266.

6 Voss S, Charrondière UR, Slimani N, Kroke A, Riboli E, Wahrendorf J, Boeing H: EPICSOFT a European computer program for 24 hour dietary protocols. Z Ernährungswiss 1998; 37:227-233

7 Bohlscheid-Thomas S, Hoting I, Boeing $\mathrm{H}$ Wahrendorf $\mathbf{J}$ : Reproducibility and relative validity of food group intake in a food frequency questionnaire developed for the German part of the EPIC Project. European Prospective Investigation into Cancer and Nutrition. Int $\mathrm{J}$ Epidemiol 1997;26(suppl 1):59-70.

8 Dehne LI, Klemm C, Henseler G, HermannKunz E: The German Food Code and Nutrient Data Base (BLS II.2). Eur J Epidemiol 1999;15: 355-359.
9 Food and Nutrition Board/Institute of Medicine: Dietary Reference Intakes for Thiamin, Riboflavin, Niacin, Vitamin $\mathrm{B}_{6}$, Folate, Vitamin $\mathrm{B}_{12}$, Pantothenic Acid, Biotin, and Choline. Washington, National Academy Press, 1998.

10 Deutsche Gesellschaft für Ernährung (DGE), Österreichische Gesellschaft für Ernährung (ÖGE), Schweizerische Gesellschaft für Ernährungsforschung (SGE), Schweizerische Vereinigung für Ernährung (SVE): Referenzwerte für die Nährstoffzufuhr. Frankfurt am Main, Umschauverlag/Braus, 2000.

11 Food and Nutrition Board/Institute of Medicine: Dietary Reference Intakes for Vitamin C, Vitamin E, Selenium, and Carotenoids. Washington, National Academy Press, 2000.

12 Klipstein-Grobusch K, Georg T, Boeing H: Interviewer variability in anthropometric measurements and estimates of body composition. Int J Epidemiol 1997;26(suppl 1):174-180.

13 Schoficld WN: Predicting basal metabolic rate, new standards and review of previous work. Hum Nutr Clin Nutr 1985;39(suppl 1):5-41.

14 Statistisches Bundesamt: Statistisches Jahrbuch 1998. Wiesbaden, Statistisches Bundesamt, 1998.

15 Willett WC: Nutritional Epidemiology. New York, Oxford University Press, 1998.

16 Voss S, Kroke A, Klipstein-Grobusch K, Boeing $\mathrm{H}$ : Is macronutrient composition of dietary intake data affected by underreporting? Results from the EPIC-Potsdam Study. European Prospective Investigation into Cancer and Nutrition. Eur J Clin Nutr 1998;52:119-126.

17 Kroke A, Klipstein-Grobusch K, Voss S, Moseneder J, Thielecke F, Noack R, Bocing H: Validation of a self-administered food-frequently questionnaire administered in the European Prospective Investigation into Cancer and Nutrition (EPIC) Study: Comparison of energy, protein, and macronutrient intakes estimated with the doubly labeled water, urinary nitrogen, and repeated 24-h dietary recall methods. Am J Clin Nutr 1999;70:439-447.
18 Slimani N, Ferrari P, Ocké M, Welch A, Boeing $\mathrm{H}$, van Liere M, Pala V, Amiano P, Lagiou A Mattisson I, Stripp C, Engeset D, Charrondière R, Buzzard M, van Staveren W, Riboli E: Standardization of the 24-hour diet recall calibration method used in the European Prospective Investigation into Cancer and Nutrition (EPIC): General concepts and preliminary results. Eur J Clin Nutr 2000;54:900-917.

19 Thiel C, Heinemann L, Thai DM: Lebensmittelaufnahme und Nährstoffversorgung in den neuen und alten Bundesländern. Ernährungsumschau 1993:40:486-490.

20 Brasche S, Winkler G, Heinrich J: Ernährungserhebungen bei Erwachsenen in Erfurt 1991/92: Nährstoffaufnahme. Z Ernährungswiss 1997;36:133-142.

21 Heseker H, Adolf T, Eberhardt W, Hartmann S, Kübler W, Schneider R: Food intake and nutrient supply in Germany - results of the VERA Study. Ernährung/Nutrition 1994;18: 158-161.

22 Ulbricht G, Schmidt G, Seppelt B: Veränderungen der Energie- und Nährstoffaufnahme der Bevölkerung im ersten Jahr der deutschen Einheit - Ergebnisse einer Modellstudie in Potsdam. Z Ernährungswiss 1996;35:150-156.

23 Thiel C, Heinemann L, Thai DM: The nutritional situation of the adult population in the new German federal states after the political changes of the early 1990s. Ernährungsforschung 1996;41:239-248.

24 Mensink GB, Thamm M, Haas K: Nutrition in Germany 1998. Gesundheitswesen 1999;61 (suppl):200-206.

25 Winkler G, Brasche S, Doring A, Heinrich J: Dietary intake of middle-aged men from an East and a West German city after the German reunification: Do differences still exist? Eur J Clin Nutr 1998;52:98-103.

26 Winkler G, Doring A, Keil U: Selected nutrient intakes of middle-aged men in southern Germany: Results from the WHO MONICA Augsburg Dietary Survey of 1984/85. Ann Nutr Metab 1991;35:284-291.

27 Hermann-Kunz E, Thamm M: Dietary recommendations and prevailing food and nutrient intakes in Germany. Br J Nutr 1999;81(suppl 2):61-69. 